\title{
p73 is essential for vitamin D-mediated osteoblastic differentiation
}

\author{
R Kommagani ${ }^{1,2}$, A Whitlatch ${ }^{1,2}$, MK Leonard ${ }^{1}$ and MP Kadakia ${ }^{*, 1}$
}

The secosteroid hormone vitamin D3 (VD3) exerts its biological actions through its cognate receptor, the vitamin D receptor (VDR). Vitamin D3 and VDR have a key function in bone formation and keratinocyte differentiation, exert antiproliferative actions in human cancer, and is widely used as a chemotherapeutic agent for cancer. In addition, VD3 promotes differentiation of human osteosarcoma cells by up-regulating genes involved in cell cycle arrest and osteoblastic differentiation. Although considerable work has been carried out in understanding the molecular mechanisms underlying the VD3-mediated differentiation of human osteosarcoma cells, the upstream regulation of VD3 signaling pathway is still unclear. In this study, we show that p73 acts as an upstream regulator of VD3-mediated osteoblastic differentiation. Transcription factor p73, a p53 homolog, has been shown to have a function in development and recently been termed as a tumor suppressor. Silencing p73 results in a significant reduction of VD3-mediated osteoblastic differentiation; although DNA damage induced p73 leads to an increase in VD3-mediated differentiation of osteosarcoma cells. Together, our data implicate a novel function for p73 in vitamin D-mediated differentiation of human osteosarcoma cells.

Cell Death and Differentiation (2010) 17, 398-407; doi:10.1038/cdd.2009.135; published online 25 September 2009

The vitamin $D$ endocrine system has a vital function in maintaining calcium and phosphate homeostasis to protect skeletal integrity. Apart from its classical functions, 1,25dihydroxyvitamin D3 (VD3), the hormonally active form of vitamin $\mathrm{D}$, also has potent antiproliferative, pro-apoptotic, and pro-differentiation actions in a number of malignances. ${ }^{1}$ Biological functions of VD3 are mediated by its binding to vitamin $\mathrm{D}$ receptor (VDR), a nuclear transcription factor that forms a heterodimer with the retinoid $X$ receptor and binds to vitamin $\mathrm{D}$ response elements (VDRE) in the promoter regions of VD3 responsive genes. ${ }^{2,3}$

The antiproliferative action of VD3 and its analogues is shown in many different human cancer cell lines, notably, colon, prostate, and breast cancer cell lines. ${ }^{4}$ Antiproliferative effects mediated by VD3 and VDR involve up-regulation of growth inhibitory genes including $p 21, p 27$, and IGFBP- $3^{5-7}$ and down-regulation of pro-proliferative genes such as $c-m y c$ and $B C L-2 .^{8}$

In addition, VD3 and its analogues also promote osteoblastic differentiation as shown by the differentiation of osteosarcoma cell lines MG-63 and SaOS2. ${ }^{9}$ Although VD3mediated osteoblastic differentiation is associated with activation of cell cycle inhibitors such as p21 and differentiation markers such as osteopontin (OPN) and osteocalcin $(\mathrm{OCN})$, the exact molecular mechanisms underlying the regulation of the VDR and VD3 signaling pathway in osteosarcoma cells is unclear. ${ }^{10-12}$ Recently, we showed that VDR is directly regulated by both $p 63$ and $p 73$, members of the p53 family. ${ }^{13,14}$ In addition, we showed that p73 is essential for the induction of VDR expression on DNA damage. ${ }^{14}$

The p73 transcription factor has been shown to have a vital function in development and human cancers. Unlike p53deficient mice, p73-deficient mice exhibit severe neurological defects, high mortality rate, and show a runting phenotype. ${ }^{15}$ Like p53, p73 also contains transactivation (TA), DNA binding, and oligomerization domains. In addition, multiple isoforms of p73 are generated because of alternative promoter usage and $3^{\prime}$ splicing. ${ }^{16}$ Full-length $\mathrm{N}$-terminal TA domain containing isoforms are termed as TA isoforms (TAp73), and the isoforms that lack the TA domain are termed as $\Delta \mathrm{N}$ isoforms $(\Delta N p 73)$. The $\Delta N p 73$ isoforms promote proliferation by inducing pro-proliferative genes and by acting in a dominant negative manner toward TAp73 isoforms and p53. ${ }^{17}$ In contrast, TAp73 isoforms are structurally and functionally similar to full-length p53 and promote cell cycle arrest and apoptosis. In particular, exogenous TAp73 isoforms are capable of inducing cell cycle arrest and apoptosis in multiple cancer cell lines. ${ }^{18}$ In addition, DNA damage mediated up-regulation of TAp73 isoforms has been shown to have a crucial function in p53-independent chemosensitivity. ${ }^{19}$ Furthermore, DNA damage mediated up-regulation of TAp73 has been shown to determine the chemosensitivity of multiple cancer cells, suggesting p73 as a potential target for cancer therapeutics. ${ }^{20}$ Herein, we investigated the function of p73 in VD3-mediated osteoblastic differentiation using SaOS2 osteosarcoma cells as a model system. Our results clearly show that p73 is essential for VD3-mediated

\footnotetext{
${ }^{1}$ Department of Biochemistry and Molecular Biology, Boonshoft School of Medicine, Wright State University, 3640 Colonel Glenn Highway, Dayton, OH 45435, USA ${ }^{*}$ Corresponding author: MP Kadakia, Department of Biochemistry and Molecular Biology, Boonshoft School of Medicine, Wright State University, 3640 Colonel Glenn Highway, 122 Diggs Laboratory, Dayton, OH 45435, USA. Tel: 937-775-2339; Fax: 937-775-3730; E-mail: madhavi.kadakia@wright.edu

${ }^{2}$ These authors contributed equally to this work.

Keywords: p73; vitamin D3; vitamin D receptor; osteosarcoma; differentiation

Abbreviations: VDR, vitamin D receptor; VDRE, vitamin D responsive elements; VD3, 1,25 dihydroxy-vitamin D3; OCN, osteocalcin; OPN, osteopontin

Received 20.2.09; revised 16.7.09; accepted 03.8.09; Edited by RA Knight; published online 25.9.09
} 
differentiation of osteosarcoma and that induction of p73 on DNA damage enhances VD3-mediated differentiation of osteosarcoma cell lines.

\section{Results}

Silencing p73 leads to a reduction in endogenous VDR expression levels in osteosarcoma. To investigate the function of p73 in VD3-mediated differentiation, we first tested whether endogenous p73 is essential for basal VDR expression. SaOS2 cells devoid of p53 were transfected with either control siRNA (con si) or two different p73 siRNAs to rule out off target effects (p73_1 si or p73_2 si). Total RNA was extracted from these cells and subjected to real-time PCR to determine the transcript levels of p73 and VDR. We observed that cells transfected with either p73_1 si or p73_2 si showed a significant reduction of basal p73 transcript levels, which correlated with a reduction in basal VDR transcript levels when compared with control siRNAtransfected cells (Figure 1a). Both the control siRNAtransfected cells and cells without any transfection (Mock) showed similar levels of p73 and VDR, thus ruling out the possibility of any nonspecific effect due to siRNA transfections (Figure 1a). Although, both siRNAs against p73 reduced the basal p73 expression levels, p73_1 si was more potent than p73_2 si, so we used p73_1 si for our subsequent studies. Consistent with the reduction in VDR transcript levels, we also observed a significant reduction in VDR protein levels in p73 siRNA-transfected cells compared with cells transfected with control siRNA as shown by immunofluorescence and western blot analyses (Figure 1b).

\section{p73 activates the osteoblastic differentiation markers} OPN and OCN through VDR. As VD3-mediated osteoblastic differentiation is associated with the transcriptional activation of OPN and OCN through VDR, we next tested whether p73 affects the basal expression of OPN and OCN through VDR. To investigate this, we first examined whether p73 silencing affects VD3-mediated transcriptional activity in SaOS2 cells. This was examined by monitoring the VD3-mediated increase in VDRE-Luc reporter activity in the presence and absence of p73. The VDRE-Luc reporter bears four VDR responsive elements upstream of a luciferase gene and is widely used for measuring VD3-mediated transcriptional activity. As expected, a significant increase in the VDRE-Luc activity was observed in cells transfected with control siRNA and treated with VD3 (Figure 2a). However, silencing p73 led to a significant reduction in VD3-mediated VDRE-Luc activity (Figure 2a). Next, we examined the transcript levels of OCN and OPN in SaOS2 cells transfected with control siRNA, p73 siRNA, or VDR siRNA (Figure 2b). As seen earlier in Figure 1a, a significant reduction in the transcript levels of p73 and VDR were observed with p73 siRNA (Figure 2b). In addition, silencing p73 also led to a significant reduction in basal OPN and $\mathrm{OCN}$ expression levels (Figure 2b). To determine whether p73 is able to induce differentiation markers OPN and OCN through VDR, we monitored the expression of OPN and OCN after silencing

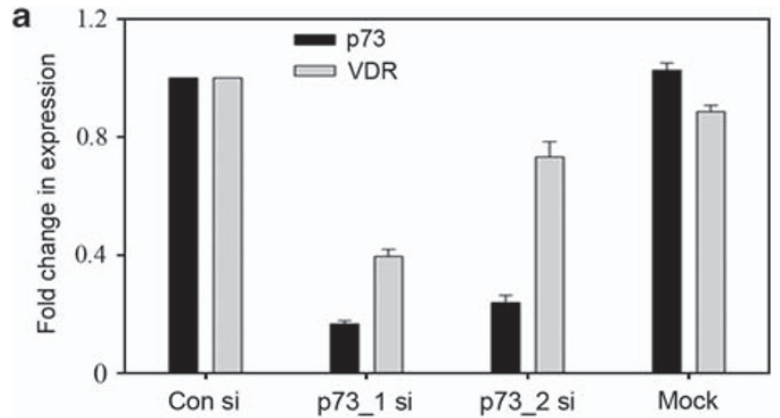

b
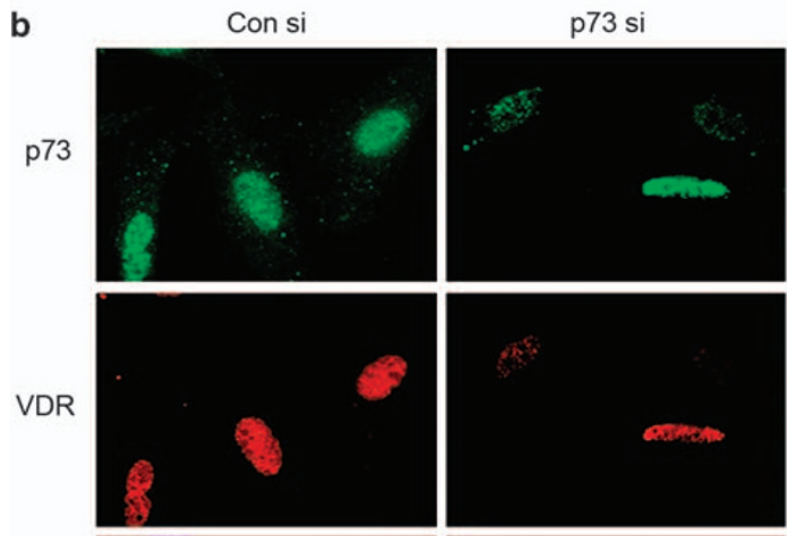

DAPI
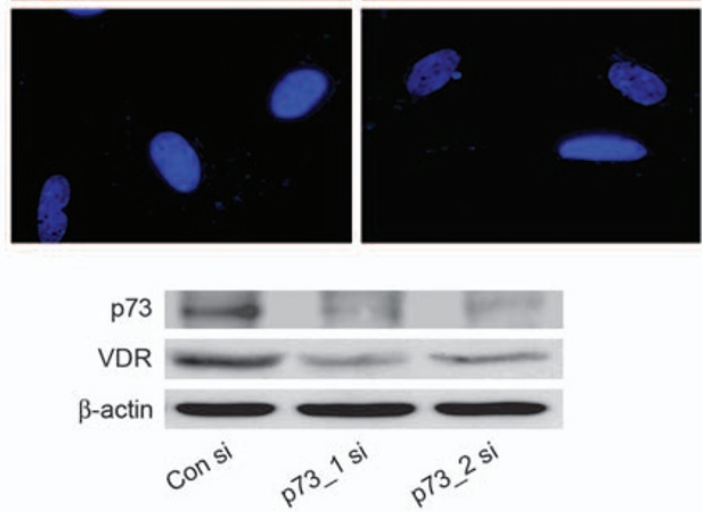

Figure 1 Silencing p73 results in reduction in VDR expression in osteosarcoma cells. (a) SaOS2 cells, mock transfected or transfected with two rounds of control siRNA or p73 siRNAs were harvested for total RNA at $48 \mathrm{~h}$ after second round of siRNA transfection. $Y$ axis represents the fold change in transcript levels of VDR and p73 in cells transfected with p73 siRNA compared with control siRNA-transfected cells determined by TaqMan-based real-time PCR. (b) SaOS2 cells were transfected with control siRNA and p73 siRNA and at $48 \mathrm{~h}$ posttransfection immunofluorescence was performed to detect the localization of p73 and VDR proteins as explained in Materials and Methods (upper panel). Whole cell extracts prepared from SaOS2 cells were transfected with control siRNA or p73 siRNA were subjected to immunoblot analysis using VDR and $\beta$-actin specific antibodies. p73 was detected by immunoprecipitation followed by western as described in Materials and Methods (lower panel)

VDR. We observed that when VDR is silenced, the expression of both OPN and OCN is reduced compared with control siRNA-treated cells (Figure $2 b$ ). This suggests that p73-mediated VDR induction is required for induction of OPN and OCN. As alkaline phosphatase has been shown to be regulated by VD3 and its activity is used as a marker for measuring the early stages of differentiation in osteoblasts, we examined whether silencing p73 affects 
alkaline phosphatase activity. We observed that upon silencing p73, the basal alkaline phosphatase activity was significantly reduced in SaOS2 cells further supporting that p73 contributes to osteoblastic differentiation (Figure 2c).

As silencing p73 led to the down-regulation in OPN and OCN, we next tested whether ectopic p73 activates VDR,
OPN, and OCN expression in SaOS2 cells. SaOS2 cells were transfected with control vector or TAp73 $\alpha$ expression plasmid and transcript levels of VDR, OCN, and OPN were monitored. We used TAp73 $\alpha$ expression for these studies, as it is the predominant isoform observed in SaOS2 cells. ${ }^{21}$ Overexpression of $\mathrm{TAp} 73 \alpha$ was confirmed by measuring the p73
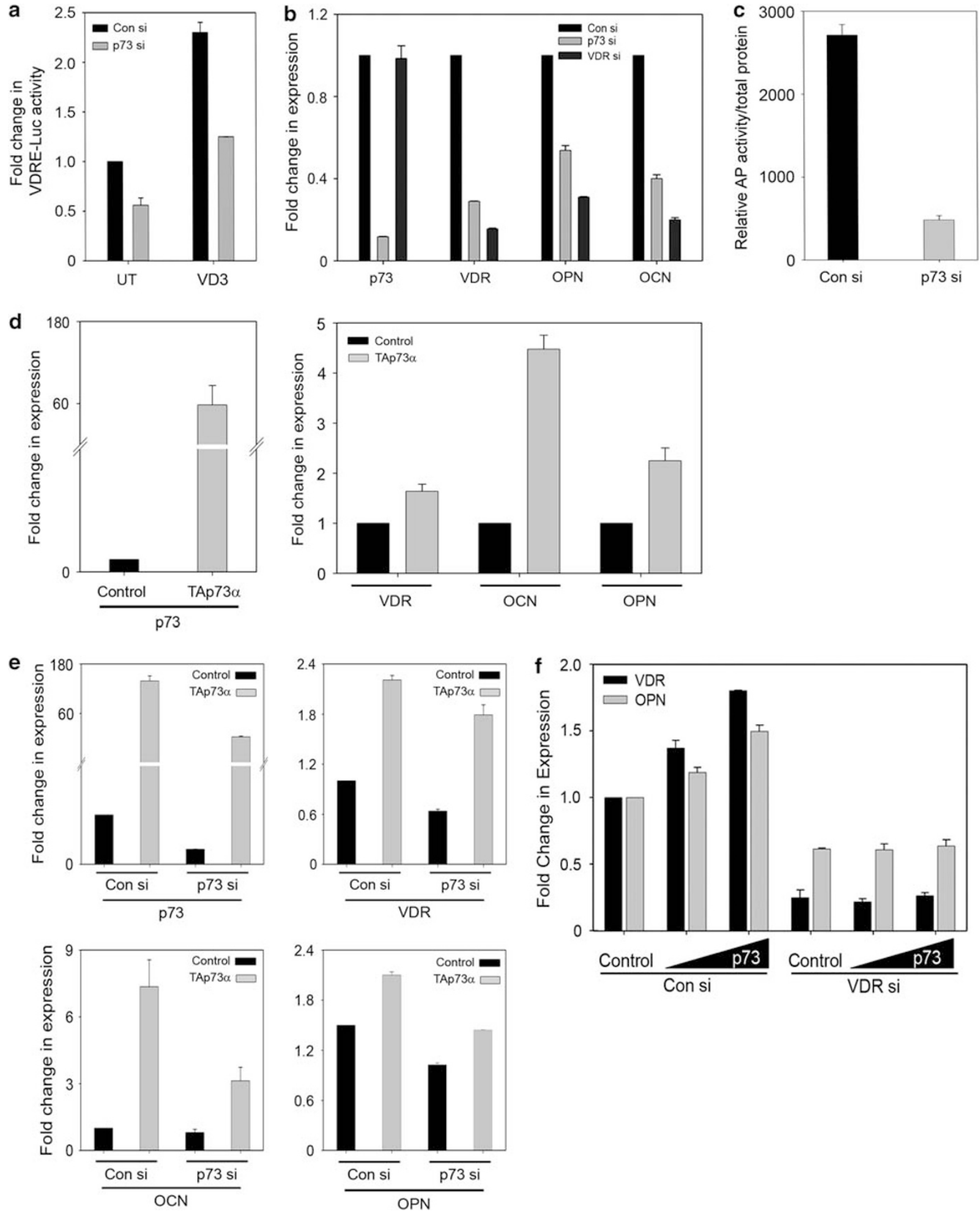
transcript (Figure 2d). A marked increase in VDR, OCN, and OPN transcripts were observed in TAp73 $\alpha$-transfected cells compared with control vector-transfected cells (Figure $2 \mathrm{~d}$ ). To confirm the specificity of TAp $73 \alpha$-mediated activation of VDR, OCN, and OPN expression, we rescued the effects of silencing $\mathrm{p} 73$ by overexpressing TAp73 $\alpha$. SaOS2 cells transfected with control siRNA or p73 siRNA were transfected with either control vector or TAp73 $\alpha$ expression plasmid and the expression of VDR, OCN, and OPN was monitored. As expected, silencing p73 led to a significant reduction of p73, VDR, OCN, and OPN transcript levels (Figure 2e). Interestingly, re-expression of TAp73 $\alpha$ in p73 siRNA-transfected cells reversed the inhibition of VDR, OCN, and OPN transcript levels observed on p73 silencing (Figure 2e). Furthermore, to unequivocally show that p73 exerts its effect on OPN and OCN expression through VDR, SaOS2 cells transfected with either con si or VDR si were transfected with increasing doses of TAp73 $\alpha$ expression (Figure 2f). We observed that cells transfected with con si followed by transfection with TAp $73 \alpha$ led to a dose-dependent increase in VDR as well OPN expression while TAp73 $\alpha$ was unable to rescue the expression of OPN in cells in which VDR was silenced. Taken together, these results suggest that in SaOS2 cells, p73 regulates the expression of OCN and OPN through VDR.

p73 is required for vitamin D-mediated osteoblastic differentiation. As VD3 and VDR primarily regulate OCN and OPN genes to promote osteoblastic differentiation and p73 knockdown also led to a decrease in basal VDR, OCN, and OPN expression levels in osteosarcoma cells, we next examined whether loss of p73 affects VD3-mediated osteoblastic differentiation. To investigate this we first studied whether VD3 has any effect on endogenous p73 in SaOS2 cells. SaOS2 cells were treated with increasing concentrations of VD3 for $48 \mathrm{~h}$, and the transcript levels of $\mathrm{p73}, \mathrm{OCN}$, and OPN were examined, the latter two were used as positive controls for VD3-mediated genomic effects. As expected, a dose-dependent induction in both OCN and OPN (Figure 3a) transcript levels were observed with increasing concentrations of VD3. We did not, however, observe a consistently significant increase in either p73 or VDR transcript on treatment with VD3, indicating that $\mathrm{p} 73$ and VDR are not transcriptionally regulated by VD3 in osteosarcoma cells (Figure 3a).
Interestingly, when effects of VD3 on p73 and VDR were studied at the protein level, we observed a slight increase in both VDR as well as p73 expression on VD3 treatment (Figure 3b). Earlier studies have shown that binding of VD3 to VDR contributes to the stabilization of VDR at the protein level, which is consistent with our findings in SaOS2 cells. ${ }^{22}$ Although these results suggest the VD3 may have a modest effect on $\mathrm{p73}$, this effect does not significantly contribute to the up-regulation seen in VDR on VD3 treatment as there were no changes in VDR transcript levels by VD3. Finally, we showed that VD3 treatment results in a dose-dependent increase in alkaline phosphatase activity of SaOS2 cells, another measure of osteoblastic differentiation (Figure 3c).

Next, to determine whether p73 has a function in VD3mediated osteoblastic differentiation of SaOS2 cells, we studied the expression of OPN and OCN in SaOS2 cells treated with VD3 in the presence and absence of p73. As expected, p73 knockdown led to a significant decrease in p73 as well as VDR transcript levels (Figure 4a). A significant increase in OCN and OPN (Figure 4a) expression levels were observed in cells transfected with control siRNA and treated with either $10 \mathrm{nM}$ or $100 \mathrm{nM}$ of VD3 for $48 \mathrm{~h}$, indicating the onset of SaOS2 cells differentiation. However, silencing p73 not only led to a decrease in VDR expression levels, but also showed a significant reduction in transcript levels of both OCN and OPN, suggesting that the basal levels of VDR, under the control of endogenous p73, are sufficient for VD3 induction of OCN and OPN (Figure 4a). In addition, we also monitored the VD3-mediated morphological changes of SaOS2 cells observed on differentiation process. We observed that cells transfected with control siRNA and treated with $10 \mathrm{nM}$ VD3 exhibited a significant number of cells with elongated cytoplasmic processes and flattened fibroblastic appearance indicative of differentiation (Figure $4 b$, marked by an arrow). However, silencing p73 led to a reduction in the number of cells with differentiation features that were observed on VD3 treatment (Figure 4b).

We next investigated whether silencing p73 affects the VD3-mediated increase in alkaline phosphatase activity. We transfected SaOS2 cells with control or p73 siRNA, and subsequently treated the cells with vehicle or VD3. As expected, cells transfected with control siRNA showed an increase in alkaline phosphatase activity when treated with VD3, which was significantly down-regulated in cells in which

Figure 2 Silencing p73 leads to a down-regulation in vitamin D transcriptional activity and VDR downstream targets. (a) SaOS2 cells transfected with either control siRNA or p73 siRNA were transfected with VDRE-Luc construct along with Renilla luciferase construct followed by treatment with $10 \mathrm{nM}$ VD3 for $48 \mathrm{~h}$. Dual luciferase assays were performed to detect the VDRE-Luc activity and $Y$ axis represents the fold change in VDRE-Luc activity compared with control siRNA-transfected cells treated with vehicle. Error bars represent standard deviation from the mean. (b) SaOS2 cells were transfected twice with either control siRNA, p73 siRNA, or VDR siRNA. Total RNA was harvested at $48 \mathrm{~h}$ and subjected to TaqMan-based real-time PCR to detect the endogenous transcript levels of VDR, p73, OPN, and OCN. $Y$ axis represents the fold change in transcript levels compared with control siRNA-transfected cells. Error bars represent standard deviation from the mean. (c) SaOS2 cells were transfected with control or p73 siRNA and after $36 \mathrm{~h}$ subjected to two freeze-thaw cycles for whole cell lysate extraction. Alkaline phosphatase activity was measured and normalized to total protein. (d) SaOS2 cells were transfected with control vector or TAp73 $\alpha$ expression plasmid and $24 \mathrm{~h}$ posttransfection transcript levels of $\mathrm{p} 73, \mathrm{VDR}, \mathrm{OCN}$, and OPN were determined. $Y$ axis represents the fold change in transcript levels of p73, VDR, OCN, and OPN compared with control vector-transfected cells. Error bars represent standard deviation from the mean. (e) SaOS2 cells were transfected twice with control siRNA and p73 siRNA and $24 \mathrm{~h}$ post-siRNA transfections, both control siRNA and p73 siRNA cells were transfected with either control vector or TAp73 $\alpha$ expression plasmid as indicated. After $24 \mathrm{~h}$, total RNA was harvested for determination of p73, VDR, OCN, and OPN transcript levels. $Y$ axis represents the fold change in transcript levels of p73, VDR, OCN, and OPN compared with control vector-transfected cells with control siRNA. Error bars represent standard deviation from the mean. (f) SaOS2 cells transfected with control siRNA or VDR siRNA were transfected with vector control or increasing amounts of TAp73 $\alpha$. At $24 \mathrm{~h}$ posttransfection, total RNA was extracted and TaqMan-based real-time PCR was performed to detect transcript levels of VDR and OPN. $Y$ axis represents the fold change in the transcript levels compared with control-treated cells 

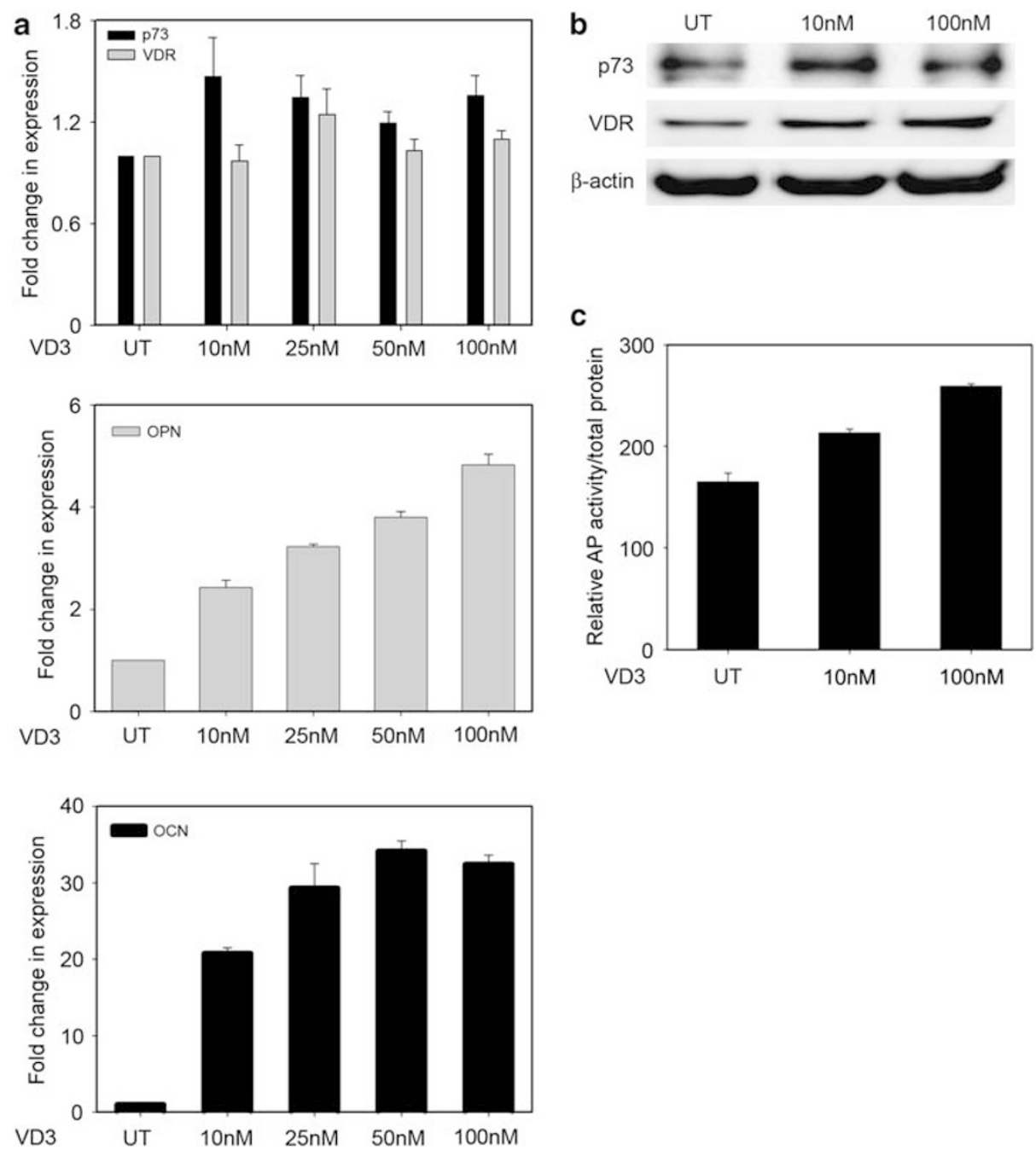

Figure 3 Vitamin D has no effect on endogenous p73 expression. (a) SaOS2 cells were treated with either vehicle (UT) or increasing concentrations of VD3 as indicated. At $48 \mathrm{~h}$ posttreatment, total RNA was extracted and TaqMan-based real-time PCR was performed to detect the transcript levels of $p 73, V D R, O P N$ and OCN. $Y$ axis represents the fold change in the transcript levels compared with vehicle-treated cells. Error bars represent standard deviation from the mean. (b) Cells were treated with vehicle or increasing concentrations of VD3 and at $48 \mathrm{~h}$ posttreatment, protein was harvested and immunoprecipitated for p73. Both immunoprecipitated and nonimmunoprecipitated samples were then subjected to western blot analysis for p73, VDR, and $\beta$-actin. (c) SaOS2 cells were treated with vehicle or two different concentrations of VD3. $Y$ axis represents the relative AP activity normalized to total protein. Error bars represent standard deviation from the mean

p73 was silenced (Figure 4c). These results clearly indicate that $\mathrm{p} 73$ is required for VD3-mediated differentiation of SaOS2 osteosarcoma cell lines.

\section{DNA damage enhances vitamin D-mediated} osteoblastic differentiation via p73. We had shown earlier that $p 73$ is essential for DNA damage induced VDR expression and subsequent enhancement of VD3 transcriptional activity in H1299, a small cell lung carcinoma cell line. ${ }^{14}$ To test whether p73-mediated induction of VDR expression levels on DNA damage can potentiate VD3-mediated differentiation of SaOS2 cells; we first verified whether DNA damage can enhance VD3mediated induction of OCN and OPN expression levels. SaOS2 cells pretreated with etoposide or doxorubicin for $24 \mathrm{~h}$ were cultured in $10 \mathrm{nM}$ VD3 for 2 days. Both VDR and p73 transcript levels were significantly induced on treatment with etoposide or doxorubicin (Figure 5a). As expected, increased transcript levels of both OPN and OCN were observed in cells treated with $10 \mathrm{nM}$ VD3 alone (Figure 5b). Interestingly, cells pre-treated with etoposide or doxorubicin and cultured in VD3 showed a further increase in both OPN and OCN expression levels when compared with VD3 treatment alone (Figure $5 b$ ). These results suggest that increased p73 and VDR expression observed on DNA damage can lead to an increase in VD3-mediated differentiation.

To confirm that p73 is essential for DNA damage-mediated enhancement in VD3-mediated differentiation, the transcript levels of OPN and OCN were examined in the presence and absence of p73 in cells pre-treated with etoposide followed by culturing in VD3. p73 silencing led to a reduction of VDR expression levels in cells treated with etoposide alone or in combination with VD3, indicating that p73 is essential for 

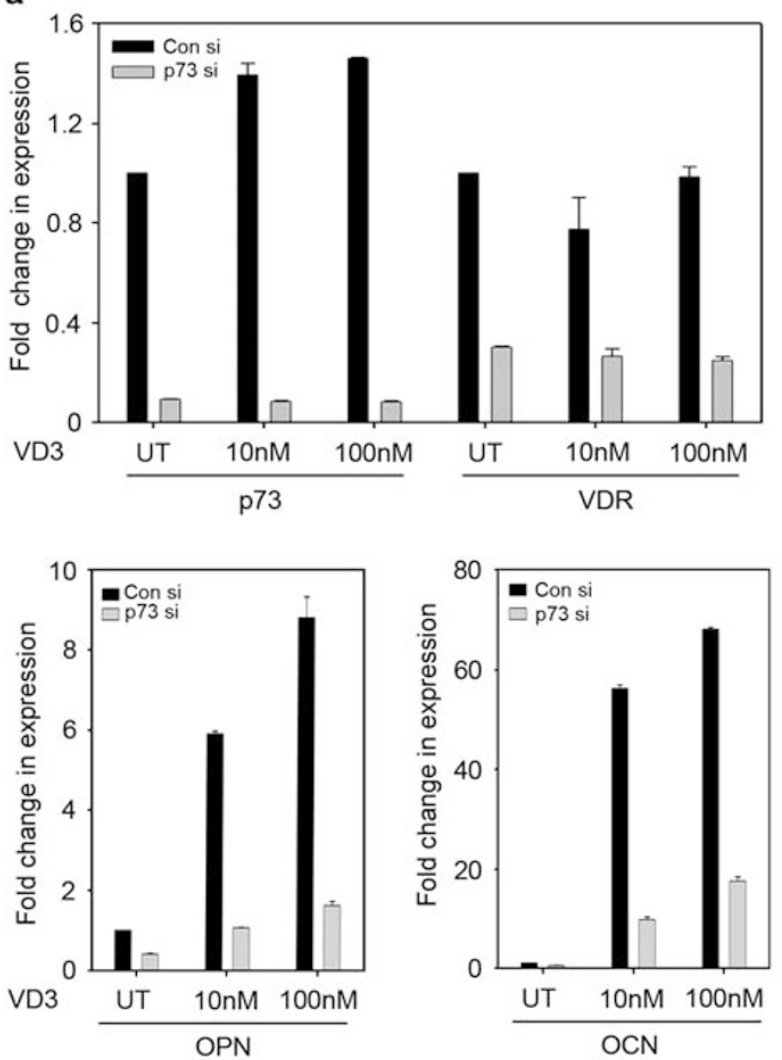

C

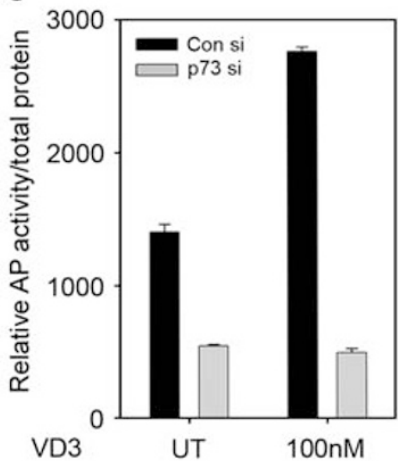

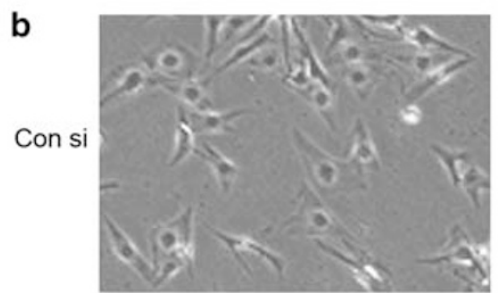

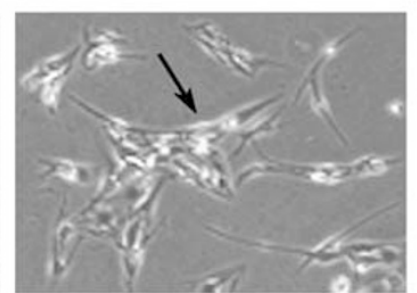

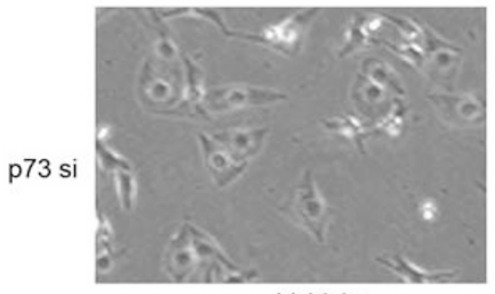

Vehicle

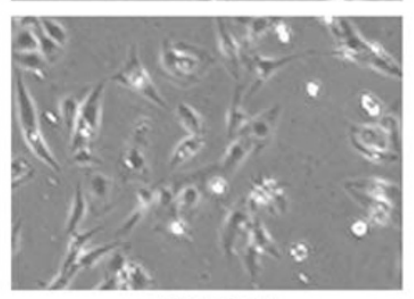

Vitamin D

Figure 4 p73 is required for vitamin D-mediated osteoblastic differentiation. (a) SaOS2 cells transfected with control siRNA or p73 siRNA were harvested and re-plated onto six-well plates. Next day, cells were treated for $48 \mathrm{~h}$ with vehicle or 10 or $100 \mathrm{nM}$ VD3 and total RNA was extracted and transcript levels of p73, VDR, OPN, and OCN were determined. $Y$ axis represents the fold change in transcript levels compared with control siRNA-transfected cells with vehicle treatment. Error bars represent standard deviation from the mean. (b) SaOS2 cells transfected with control siRNA or p73 siRNA were split and re-plated onto six wells. Next day, cells were treated with vehicle or $10 \mathrm{nM} \mathrm{VD3}$ as indicated and at $48 \mathrm{~h}$ post-VD3 treatment morphological changes in cells were observed by phase contrast microscopy. (c) SaOS2 cells were transfected with control or p73 siRNA and subsequently treated with vehicle or $100 \mathrm{nM}$ VD3 for $24 \mathrm{~h}$. Whole cell lysates were then collected and alkaline phosphatase activity was measured. $Y$ axis represents the relative AP activity normalized to total protein

induction of VDR on DNA damage in these cells (Figure 6a). Silencing p73 also led to a significant decrease in OPN and OCN expression levels in cells treated with VD3 alone or in combination with etoposide (Figure 6b). In addition, cells transfected with p73 siRNA showed a reduced VDR expression at the protein level compared with control siRNA-treated cells in the absence of etoposide (Figure 6a, right panel).
However, etoposide-treated cells even in the presence of p73 siRNA showed considerable levels of VDR protein, suggesting that stabilized p73 protein upon DNA damage can induce VDR expression (Figure 6a, right panel). Together, these results clearly indicate that induction of VDR by $\mathrm{p73}$ on DNA damage can enhance VD3-mediated differentiation of SaOS2 cells. 

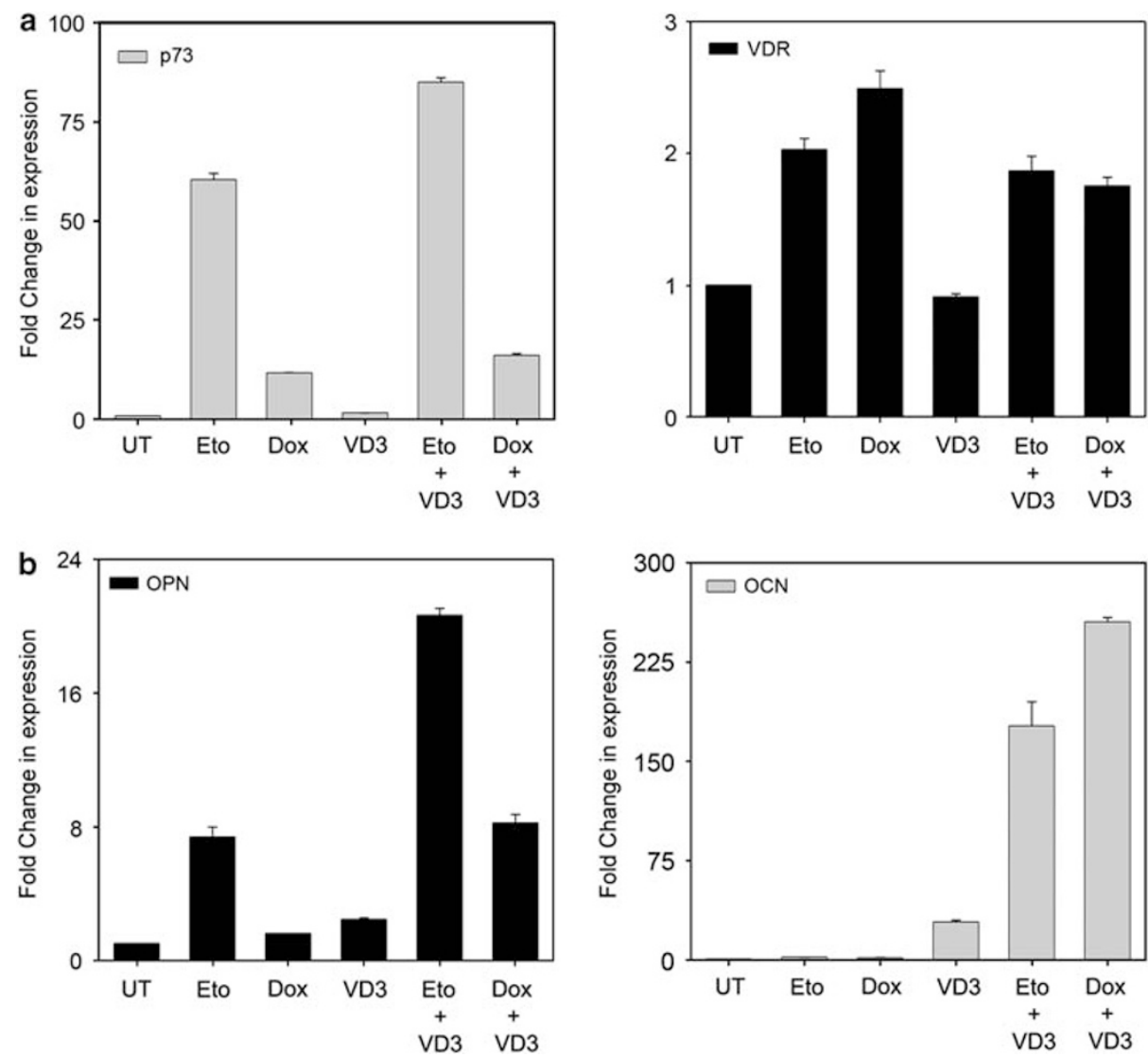

Figure 5 DNA damage enhances vitamin D-mediated osteoblastic differentiation. (a) SaOS2 cells were pretreated for $24 \mathrm{~h}$ with $4 \mu \mathrm{M}$ etoposide or $0.4 \mu \mathrm{M}$ doxorubicin and subsequently cultured with vehicle or $10 \mathrm{nM}$ VD3 for an additional $48 \mathrm{~h}$. Total RNA was extracted and transcript levels of p73 and VDR (a) and OPN and OCN (b) were determined by TaqMan-based real-time PCR. Y axis represents the fold change in transcript levels compared with vehicle-treated (UT) cells. Error bars represent standard deviation from the mean

\section{Discussion}

Osteoblasts are the main bone forming cells, which are essential for deposition of bone extracellular matrix. Osteoblasts are derived from pluripotent mesenchymal cells during embryonic development. A characteristic feature of osteoblasts is that they can be cultured in vitro to proliferate and differentiate. ${ }^{23}$ Osteoblastic differentiation is essential for proper bone formation and is mediated by multiple factors including VDR. ${ }^{24}$ The function of the VD3 in bone formation and in osteoblastic differentiation is well documented. ${ }^{25}$ Vitamin D3 has been shown to promote differentiation of mature osteoblasts through the regulation of osteoblastassociated genes including OCN and OPN ${ }^{26}$ Osteosarcomas are the most common type of bone cancers, retaining characteristics of primary osteoblasts and are widely used for studying osteoblasts. ${ }^{27}$ Vitamin D3 and its analogues have been shown to promote the differentiation of osteosarcoma cells. ${ }^{28,29}$ Although, VD3 exerts its biological functions on osteoblasts or osteosarcoma cells, the upstream regulation of the VD3 pathway in osteoblasts is still understudied. In this study, we showed that silencing p73 resulted in the downregulation of both basal VDR expression and VD3-mediated transcriptional activity in SaOS2 osteosarcoma cells (Figures 1 and 2). In SaOS2 cells, ectopic TAp73 $\alpha$ induced the expression of VDR, OCN, and OPN whereas silencing endogenous p73 led to a significant down-regulation in VDR, OCN, and OPN expression levels, which were rescued by p73 overexpression (Figure 2). These results show that in osteosarcoma cells, p73 modulates OCN and OPN expression by activating VDR.

Furthermore, down-regulation of $p 73$ resulted in significant down-regulation in VD3-mediated induction of OPN and OCN and the differentiation of SaOS2 cells (Figure 4). These results suggest a novel upstream regulation of the vitamin $D$ signaling pathway by $\mathrm{p} 73$ in osteosarcoma cells. Transforming growth factor $\beta$ (TGF- $\beta$ ) has also been shown to have a vital function in bone remodeling. ${ }^{30}$ In particular, TGF- $\beta$ has been shown to promote the proliferation of primary osteoblasts as well as shown to suppress the VD3-mediated induction of OCN. ${ }^{31}$ Interestingly, transcriptional repression of TAp73 by TGF- $\beta$ has been reported in transformed keratinocytes. ${ }^{32}$ Thus, it is likely that TGF- $\beta$ inhibits the VD3-mediated differentiation of osteoblasts by repressing $p 73$. Furthermore, given both VDR and $\mathrm{p} 73$ null mice are runted in phenotype, it will be interesting to see whether regulation of VD3 functions by $p 73$ is essential for bone formation during embryonic development. ${ }^{15,25}$

Vitamin D3 and its analogues exert antiproliferative actions in different cancer cells through VDR. As expression of p73 

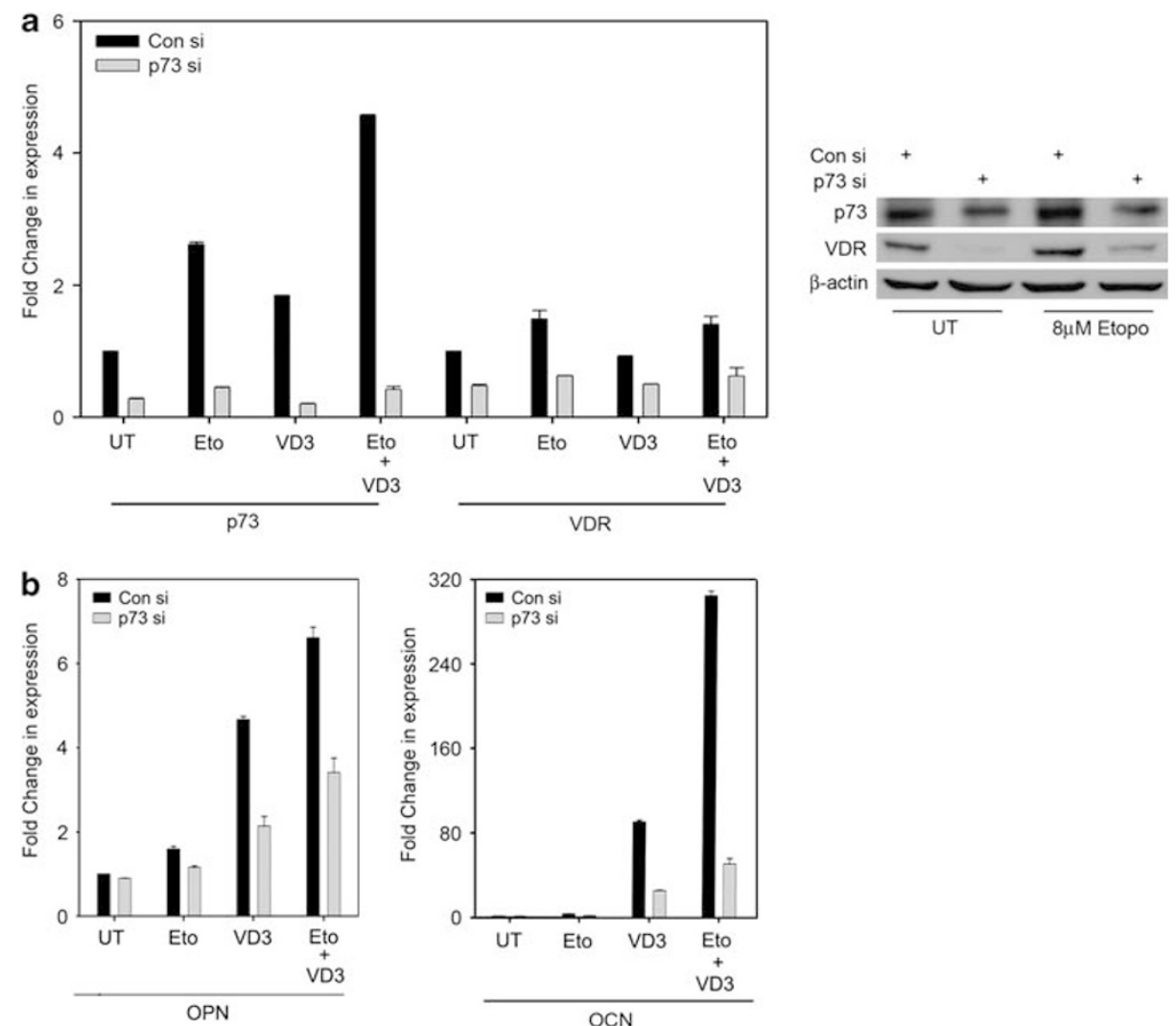

Figure 6 DNA damage potentiates vitamin D-mediated differentiation via p73. (a) SaOS2 cells were transfected with either control siRNA or p73 siRNA as indicated and treated with $4 \mu \mathrm{M}$ etoposide or left untreated as indicated. Next day, cells were either cultured in vehicle or $10 \mathrm{nM}$ VD3 for additional $48 \mathrm{~h}$ as indicated. Transcript levels of p73 and VDR were determined. Total protein was extracted and subjected to immunoprecipitation for p73 detection or to standard western blot analysis for VDR and $\beta$-actin. (b) Transcript levels of OPN and OCN were determined as in (a) after siRNA, drug and VD3 treatment. $Y$ axis represents the fold change in transcript levels compared with control siRNA-transfected cells treated with vehicle. Error bars represent standard deviation from the mean

has been reported in the majority of human cancer cells, results from our study warrant further investigation into whether p73 is essential for VDR expression and subsequently for VD3-mediated biological functions in all VD3 responsive human cancer cell lines. ${ }^{33}$ Interestingly, adenoviral-based transfer of p53 family members has been shown to promote apoptosis and cell cycle arrest in multiple cancer cell lines. ${ }^{34,35}$ Therefore, adenoviral-based gene therapy of p73 in combination with vitamin $D$ could be used as an effective therapeutic strategy against some human cancers including osteosarcomas.

Combinatorial use of VD3 and its analogues with chemotherapeutic agents have been shown to be more potent in promoting growth inhibition than using them alone. ${ }^{24,36,37}$ Clinical trials are currently underway testing the combinatorial use of VD3 analogues and chemotherapeutic agents for cancer chemotherapy. ${ }^{38,39}$ Interestingly, a wide range of chemotherapeutic agents have been shown to induce the p73 expression levels in multiple cancer cells. ${ }^{20,40}$ Results from our study showed that the regulation of VDR by $p 73$ on DNA damage can potentiate VD3-mediated differentiation (Figures 5 and 6). Thus, these findings show a novel upstream regulation of the vitamin $D$ signaling pathway by $p 73$ and provide evidence for the requirement of $p 73$ for VD3-mediated osteoblastic differentiation. Taken together, results from this study provide an insight into the combinatorial use of chemotherapeutic agents with vitamin D3 for cancer treatment, in particular, oseosarcoma.

\section{Materials and Methods}

Cell lines and sequences for siRNA. Human osteosarcoma cell line SaOS2 was obtained from ATCC and maintained in Dulbecco's modified eagle medium with $10 \%$ fetal bovine calf serum and $250 \mathrm{U}$ of Penicillin and $250 \mu \mathrm{g}$ of Streptomycin (PS). Sequences of siRNA oligonucleotides purchased from Qiagen (Qiagen, Vlencia, CA, USA) were p73_1 siRNA, sense CGGGAUGCUCAACAACC AUtt and antisense rAUGGUUGUUGAGCAUCCCGgg and p73_2 siRNA, sense CGUGACCGACGUCGUGAAAtt and antisense gtGCACUGGCUGCAGCACUUU. Sequences used for VDR siRNA are as follows, Sense $5^{\prime}$-GCGUCAGUGACGU GACCAAtt-3' and antisense: $3^{\prime}$ ggCGCAGUCACUGCACUGGUU $-5^{\prime}$.

RNA extraction and RT-qPCR analysis. Total RNA from cells was extracted by using eZNA RNA isolation kit (Omega Bio-tek, Norcross, GA, USA) and $1 \mu \mathrm{g}$ of total RNA was used to synthesize CDNA by using TaqMan reverse transcription kit (Applied Biosystems, Foster City, CA, USA). Quantitative real-time $\mathrm{PCR}$ analysis was performed by using TaqMan $2 \mathrm{X}$ master mix and assays on demand specific for VDR (Hs_0017213_m1), p73 (Hs_ 00232088_m1), OPN (Hs_00167093_m1), and OCN (Hs_01587813_m1) (PE Applied Biosystems).

Calcium phosphate-based transfections for siRNA and TA assays. For siRNA transfection, cells were seeded before the day of 
transfection and at around 30-40\% confluency and siRNA oligonucleotides were transfected by using calcium phosphate method as described earlier. ${ }^{14}$ After $24 \mathrm{~h}$, another round of siRNA transfections were carried out, and after $48 \mathrm{~h}$, cells were processed for further analysis. For VDRE-Luc TA assays, SaOS2 cells transfected with either control siRNA or p73 siRNA were subsequently transfected with $250 \mathrm{ng}$ of VDRE-Luc construct along with Renilla luciferase construct using Lipofectamine 2000 (Invitrogen, Carlsbad, CA, USA). At $6 \mathrm{~h}$ posttransfection, cells were treated with vehicle control (UT) or $100 \mathrm{nM}$ VD3 in charcoal-treated media. At $48 \mathrm{~h}$, cells were harvested in PBS lysis buffer and dual luciferase assays were performed as per manufacture protocol (Promega, Madison, WI, USA).

Immunofluorescence assay. SaOS2 cells were grown on coverslips and transfected with either control siRNA or p73 siRNA and $48 \mathrm{~h}$ posttransfection cells were subjected to an immunofluorescence assay. Briefly, cells were fixed with $2 \%$ paraformaldehyde and permeabilized in $0.2 \%$ Triton-X detergent. Cells were washed and blocked in PBS with $0.5 \%$ normal goat serum then incubated with mouse monoclonal anti-VDR D-6 (Santa Cruz Biotechnology, Santa Cruz, CA, USA) and rabbit polyclonal anti-p73 A300 (Bethyl Laboratories Inc., Montgomery, TX, USA) antibodies. Subsequently, cells were subjected to dual immunostaining using Texas Red-conjugated anti-mouse and FITC-conjugated anti-rabbit antibodies (Jackson Laboratories, PA, USA) and nuclei were visualized by 4,6diamidino-2-phenylindole. Images were captured using a Leica 6000B inverted microscope.

Western blot and immunoprecipitation. Whole cell lysates were prepared by lysing the cells in a high-salt buffer $(300 \mathrm{mM} \mathrm{NaCl}, 100 \mathrm{mM}$ Tris $\mathrm{pH}=8,0.2 \mathrm{mM}$ EDTA, $0.1 \% \mathrm{NP} 40,10 \%$ glycerol) to enrich for nuclear proteins. Total protein amounts were measured using BCA (Pierce, Rockford, IL, USA) protein detection method and equivalent amounts of protein extracts were fractionated by $10 \%$ SDS-PAGE and transferred onto polyvinylidene difluoride membranes and blocked with $5 \%$ blocking milk solution. Membranes were subsequently immunoblotted with antibodies to detect specific proteins. Monoclonal anti-VDR D-6 (Santa Cruz Biotechnology) and monoclonal anti- $\beta$-actin (Sigma, St. Louis, MO, USA) antibodies were used to detect VDR and $\beta$-actin expression, respectively. Appropriate horseradish peroxidase-conjugated secondary antibodies (Promega) were used for chemiluminescence detection with Supersignal Westpico Chemiluminescent Substrate kit (Pierce).

For immunoprecipitation studies, SaOS2 cell extracts were harvested as described above and $1 \mathrm{mg}$ of protein was diluted to a final concentration of $100 \mathrm{mM}$ $\mathrm{NaCl}$. This was precleared with $25 \mu$ l of Protein G-Sepharose beads for an hour followed by incubation with $1 \mu \mathrm{g}$ each of $\mathrm{p} 73$ monoclonal antibodies Ab-4 (Thermo Fisher Scientific Inc., Fremont, CA, USA) and IMG-259 (Imgenex, San Diego, CA, USA) for $1 \mathrm{~h}$ at $4{ }^{\circ} \mathrm{C}$. Protein $\mathrm{G}$-Sepharose beads were added again and the mixture incubated an additional $2 \mathrm{~h}$ at $4{ }^{\circ} \mathrm{C}$. The beads were pelleted by centrifugation, and washed three times with $100 \mathrm{mM} \mathrm{NaCl}$ buffer $(100 \mathrm{mM} \mathrm{NaCl}, 100 \mathrm{mM}$ Tris $\mathrm{pH}=8$, $0.2 \mathrm{mM}$ EDTA, $0.1 \%$ NP40, $10 \%$ glycerol). The isolated beads were resuspended in $70 \mathrm{uL} 100 \mathrm{mM} \mathrm{NaCl}$ buffer, boiled for $5 \mathrm{~min}$ at $90^{\circ} \mathrm{C}$ and resolved by western blot analysis as described above with rabbit polyclonal anti-p73 A300 antibody (Bethyl Laboratories).

Alkaline phosphatase assay. Alkaline phosphatase activity was performed as described earlier. ${ }^{41}$ Briefly, cells were washed with PBS and lysed with $0.1 \%$ Triton X-100 (Sigma). The lysates were then subjected to two freeze-thaw cycles and cleared through centrifugation. A measure of $2 \mathrm{mg} / \mathrm{ml}$ of $p$-nitrophenol phosphate in an alkaline buffer $(\mathrm{pH}=8)$ (Sigma) was added to the lysates and the reaction stopped by adding $20 \mathrm{mM} \mathrm{NaOH}$. The conversion of $p$-nitrophenol phosphate to $p$-nitrophenol was measured at $405 \mathrm{nM}$ (Safire ${ }^{2}$, Tecan, San Jose, CA, USA) and normalized to total protein.

Acknowledgements. We thank Stefanie Lewis for technical assistance. This work was supported by a grant from the NCI/NIH (CA118315-2) to MK.

1. Bikle DD. Vitamin D and skin cancer. J Nutr 2004; 134 (12 Suppl): 3472S-3478S.

2. Cooke NE, Haddad JG. Vitamin D binding protein (Gc-globulin). Endocr Rev 1989; 10: 294-307.

3. Nezbedova P, Brtko J. 1alpha,25-dihydroxyvitamin D3 inducible transcription factor and its role in the vitamin D action. Endocr Regul 2004; 38: 29-38.
4. Campbell MJ, Adorini L. The vitamin D receptor as a therapeutic target. Expert Opin Ther Targets 2006; 10: 735-748.

5. Bouillon R, Eelen G, Verlinden L, Mathieu C, Carmeliet G, Verstuyf A. Vitamin D and cancer. J Steroid Biochem Mol Biol 2006; 102: 156-162.

6. Huang YC, Chen JY, Hung WC. Vitamin D3 receptor/Sp1 complex is required for the induction of p27Kip1 expression by vitamin D3. Oncogene 2004; 23: 4856-4861.

7. Liu M, Lee MH, Cohen M, Bommakanti M, Freedman LP. Transcriptional activation of the Cdk inhibitor p21 by vitamin D3 leads to the induced differentiation of the myelomonocytic cell line U937. Genes Dev 1996; 10: 142-153.

8. Banerjee $P$, Chatterjee M. Antiproliferative role of vitamin $D$ and its analogs-a brief overview. Mol Cell Biochem 2003; 253: 247-254.

9. Finch JL, Dusso AS, Pavlopoulos T, Slatopolsky EA. Relative potencies of 1,25$(\mathrm{OH})(2) \mathrm{D}(3)$ and 19-Nor-1,25-(OH)(2)D(2) on inducing differentiation and markers of bone formation in MG-63 cells. J Am Soc Nephrol 2001; 12: 1468-1474.

10. Paredes R, Arriagada G, Cruzat F, Olate J, Van Wijnen A, Lian J et al. The Runx2 transcription factor plays a key role in the 1alpha,25-dihydroxy Vitamin D3-dependent upregulation of the rat osteocalcin $(\mathrm{OC})$ gene expression in osteoblastic cells. J Steroid Biochem Mol Biol 2004; 89-90: 269-271.

11. Shen $Q$, Christakos $S$. The vitamin $D$ receptor, Runx2, and the Notch signaling pathway cooperate in the transcriptional regulation of osteopontin. J Biol Chem 2005; 280: 40589-40598.

12. Zenmyo M, Komiya S, Hamada T, Hiraoka K, Kato S, Fujii T et al. Transcriptional activation of p21 by vitamin $D(3)$ or vitamin $K(2)$ leads to differentiation of p53-deficient MG-63 osteosarcoma cells. Hum Pathol 2001; 32: 410-416.

13. Kommagani R, Caserta TM, Kadakia MP. Identification of vitamin D receptor as a target of p63. Oncogene 2006; 25: 3745-3751.

14. Kommagani R, Payal V, Kadakia MP. Differential regulation of vitamin D receptor (VDR) by the p53 Family: p73-dependent induction of VDR upon DNA damage. J Biol Chem 2007; 282: 29847-29854.

15. Yang A, Walker N, Bronson R, Kaghad M, Oosterwegel M, Bonnin J et al. p73-deficient mice have neurological, pheromonal and inflammatory defects but lack spontaneous tumours. Nature 2000; 404: 99-103.

16. Kaghad M, Bonnet H, Yang A, Creancier L, Biscan JC, Valent A et al. Monoallelically expressed gene related to $\mathrm{p} 53$ at $1 \mathrm{p} 36$, a region frequently deleted in neuroblastoma and other human cancers. Cell 1997; 90: 809-819.

17. Stiewe T, Zimmermann S, Frilling A, Esche $H$, Putzer BM. Transactivation-deficient DeltaTA-p73 acts as an oncogene. Cancer Res 2002; 62: 3598-3602.

18. Melino G, De Laurenzi V, Vousden KH. p73: friend or foe in tumorigenesis. Nat Rev Cancer 2002; 2: 605-615.

19. Lissy NA, Davis PK, Irwin M, Kaelin WG, Dowdy SF. A common E2F-1 and p73 pathway mediates cell death induced by TCR activation. Nature 2000; 407: 642-645.

20. Irwin MS, Kondo K, Marin MC, Cheng LS, Hahn WC, Kaelin Jr WG. Chemosensitivity linked to p73 function. Cancer Cell 2003; 3: 403-410.

21. Chen $X$, Zheng $Y$, Zhu J, Jiang J, Wang J. p73 is transcriptionally regulated by DNA damage, p53, and p73. Oncogene 2001; 20: 769-774.

22. Healy KD, Frahm MA, DeLuca HF. 1,25-Dihydroxyvitamin D3 up-regulates the renal vitamin $D$ receptor through indirect gene activation and receptor stabilization. Arch Biochem Biophys 2005; 433: 466-473.

23. Stein GS, Lian JB, Stein JL, Van Wijnen AJ, Montecino M. Transcriptional control of osteoblast growth and differentiation. Physiol Rev 1996; 76: 593-629.

24. St-Arnaud R. The direct role of vitamin D on bone homeostasis. Arch Biochem Biophys 2008; 473: 225-230.

25. Yoshizawa T, Handa Y, Uematsu Y, Takeda S, Sekine K, Yoshihara Y et al. Mice lacking the vitamin $D$ receptor exhibit impaired bone formation, uterine hypoplasia and growth retardation after weaning. Nat Genet 1997; 16: 391-396.

26. Matsumoto T, Igarashi $\mathrm{C}$, Takeuchi $\mathrm{Y}$, Harada S, Kikuchi T, Yamato $\mathrm{H}$ et al. Stimulation by 1,25-dihydroxyvitamin D3 of in vitro mineralization induced by osteoblast-like MC3T3-E1 cells. Bone 1991; 12: 27-32.

27. Gill RK, Bell NH. Steroid receptor co-activator-1 mediates 1,25-dihydroxyvitamin $D(3)$ stimulated alkaline phosphatase in human osteosarcoma cells. Calcif Tissue Int 2000; 66: 370-374.

28. Matsumoto T, Sowa Y, Ohtani-Fujita N, Tamaki T, Takenaka T, Kuribayashi K et al. p53independent induction of WAF1/Cip1 is correlated with osteoblastic differentiation by vitamin D3. Cancer Lett 1998; 129 (1): 61-68.

29. Barroga EF, Kadosawa T, Okumura M, Fujinaga T. Effects of vitamin D and retinoids on the differentiation and growth in vitro of canine osteosarcoma and its clonal cell lines. Res Vet Sci 1999; 66: 231-236.

30. Centrella M, McCarthy TL, Canalis E. Transforming growth factor-beta and remodeling of bone. J Bone Joint Surg Am 1991; 73: 1418-1428.

31. Kassem M, Kveiborg M, Eriksen EF. Production and action of transforming growth factorbeta in human osteoblast cultures: dependence on cell differentiation and modulation by calcitriol. Eur J Clin Invest 2000; 30: 429-437.

32. Waltermann A, Kartasheva NN, Dobbelstein M. Differential regulation of p63 and p73 expression. Oncogene 2003; 22: 5686-5693.

33. Muller M, Schleithoff ES, Stremmel W, Melino G, Krammer PH, Schilling T. One, two, three-p53, p63, p73 and chemosensitivity. Drug Resist Updat 2006; 9: 288-306. 
34. Das S, Nama S, Antony S, Somasundaram K. p73 beta-expressing recombinant adenovirus: a potential anticancer agent. Cancer Gene Ther 2005; 12: 417-426.

35. Kunisaki R, Ikawa S, Maeda T, Nakazaki Y, Kurita R, Harata M et al. p51/p63, a novel p53 homologue, potentiates p53 activity and is a human cancer gene therapy candidate. J Gene Med 2006; 8: 1121-1130.

36. Hershberger PA, McGuire TF, Yu WD, Zuhowski EG, Schellens JH, Egorin MJ et al. Cisplatin potentiates 1,25-dihydroxyvitamin D3-induced apoptosis in association with increased mitogen-activated protein kinase kinase kinase 1 (MEKK-1) expression. Mol Cancer Ther 2002; 1: 821-829.

37. Moffatt KA, Johannes WU, Miller GJ. 1Alpha,25dihydroxyvitamin D3 and platinum drugs act synergistically to inhibit the growth of prostate cancer cell lines. Clin Cancer Res 1999; 5: 695-703.
38. Trump DL, Potter DM, Muindi J, Brufsky A, Johnson CS. Phase II trial of high-dose, intermittent calcitriol (1,25 dihydroxyvitamin D3) and dexamethasone in androgenindependent prostate cancer. Cancer 2006; 106: 2136-2142.

39. Fakih MG, Trump DL, Muindi JR, Black JD, Bernardi RJ, Creaven PJ et al. A phase I pharmacokinetic and pharmacodynamic study of intravenous calcitriol in combination with oral gefitinib in patients with advanced solid tumors. Clin Cancer Res 2007; 13: 1216-1223.

40. Urist M, Tanaka T, Poyurovsky MV, Prives C. p73 induction after DNA damage is regulated by checkpoint kinases Chk1 and Chk2. Genes Dev 2004; 18: 3041-3054.

41. Ciovacco WA, Goldberg CG, Taylor AF, Lemieux JM, Horowitz MC, Donahue HJ et al. The role of gap junctions in megakaryocyte-mediated osteoblast proliferation and differentiation. Bone 2009; 44: 80-86. 